\title{
Prevalence and clinical features of selective immunoglobulin A deficiency in coeliac disease: an Italian multicentre study
}

F Cataldo, V Marino, A Ventura, G Bottaro, G R Corazza, and the Italian Society of Paediatric Gastroenterology and Hepatology (SIGEP), and "Club del Tenue" Working Groups on Coeliac Disease

\begin{abstract}
Background-Selective immunoglobulin A (IgA) deficiency (SIgAD) is associated with coeliac disease (CD).

Aim-To make a retrospective study of the association of SIgAD with CD in Italy. Methods-Hospital medical records of 2098 patients consecutively diagnosed as having CD were reviewed.

Results-Of 2098 patients with CD, 54 (2.6\%) had SIgAD, representing a 10-16fold increase over that in the population in general. This increase was not influenced by age or geographical factors. Patients with SIgAD had a higher incidence of silent forms $(7 / 54,13 \%)$, recurrent infections $(16 / 54,29.6 \%)$, and atopic diseases (7/54, 13\%) than those without. The association with autoimmune and malignant diseases and the outcome after eating a gluten free diet were similar in patients with or without SIgAD. In all patients with SIgAD, antibodies for IgA gliadin and endomysium were absent, but serum levels of IgG anti-gliadin antibodies were high in almost all of them (51/54).

Conclusions-Serum IgA should be measured in order to be able to interpret negative results for IgA anti-gliadin antibodies and anti-endomysial antibodies in patients being screened for CD. Since some patients with CD and SIgAD may be negative for IgG anti-gliadin antibodies, an intestinal biopsy should be performed in all suspected cases.

(Gut 1998;42:362-365)
\end{abstract}

Keywords: coeliac disease; IgA deficiency

Selective immunoglobulin A (IgA) deficiency (SIgAD) is associated with coeliac disease (CD) and has greater prevalence than in the general population. ${ }^{1-4}$ SIgAD may cause a predisposition to autoimmune diseases and to recurrent infections in patients with $\mathrm{CD}$ and without, but to date there are few reports on the epidemiology, genetics, and clinical consequences of this association. ${ }^{1-6}$

This paper describes a multicentre retrospective study on the association between SIgAD and CD which was carried out under the auspices of the Italian Society of Paediatric Gastroenterology and Hepatology (SIGEP) and the "Club del Tenue".

\section{Patients and methods}

This was a nationwide study, with the collaboration of 25 adult and paediatric gastroenterology centres. It was retrospective, reviewing the hospital medical records of 2098 patients consecutively diagnosed as having CD from January 1990 to December 1995; the median follow up period was three years (range one to five). Both original $^{7}$ and revised ${ }^{8}$ criteria for the diagnosis of CD were used.

Immunoglobulins were measured in all the patients, and those with serum IgA concentrations below $0.05 \mathrm{~g} / \mathrm{l}$ in the presence of normal levels of serum IgG and IgM were regarded as being SIgAD; those with serum IgA concentrations lower than two standard deviations according to age were regarded as being partially IgA deficient. None of the patients were taking phenytoin, penicillamine, or sulphasalazine, agents known to induce secondary IgA deficiency. ${ }^{9}$ The control group consisted of CD patients with normal serum IgA, diagnosed in the same period and in the same centres using the same criteria.

Eight of the participating centres, with 763 patients with CD, were in Northern Italy (Brescia, Milano I, Milano II, Milano III, Padova, Torino I, Torino II, Trieste), ten with 562 patients were in Central Italy (Ancona, Bologna I, Bologna II, Chieti, Imola, Modena, Perugia, Pisa, Roma I, Roma II), and seven with 773 patients were in Southern Italy (Bari, Catania, Catanzaro, Napoli I, Napoli II, Palermo, S Giovanni Rotondo).

The age distribution of the patients was as follows: 1776 were children (aged from 6 months to 15 years; 745 males and 1031 females) and 322 were adults (aged from 18 to 65 years; 122 men and 200 women).

From the clinical histories of the patients with or without SIgAD we recorded: (a) clinical findings of CD at diagnosis; (b) associated diseases at diagnosis and during follow up; (c) early feeding practices in the children; (d) mucosal lesions at diagnosis and after a gluten free diet (GFD); (e) laboratory findings at diagnosis and after GFD; (f) clinical course after GFD.

In each centre, serum immunoglobulin levels (IgA, IgM, IgG) were assayed by nephelometry, ${ }^{10}$ and anti-gliadin antibodies (AGAs) of both IgA and IgG classes were measured by enzyme linked immunoassay (Alfagliatest; Eurospital, Trieste, Italy). Antiendomysial antibodies (EMAs) were deter-
Dr Cataldo.

Accepted for publication 5 September 1997 
Table 1 Clinical pattern in patients with selective $\operatorname{Ig} A$ deficiency (SIgAD) and in those with normal serum $\operatorname{Ig} A$

\begin{tabular}{|c|c|c|c|c|}
\hline & $\begin{array}{l}\text { A Patients with } \\
\text { SIgAD }(n=54) \\
(\%)\end{array}$ & $\begin{array}{l}\text { B Patients with } \\
\text { normal IgA }(n= \\
\text { 2044) (\%) }\end{array}$ & $\begin{array}{l}\text { CAdults with } \\
\text { SIgAD }(n=8) \\
(\%)\end{array}$ & $\begin{array}{l}D \text { Children with } \\
\text { SIgAD }(n=46) \\
(\%)\end{array}$ \\
\hline Classic forms $\dagger$ & $31(57)$ & $1306(63.9)$ & $6(75)$ & $25(54.3)$ \\
\hline Atypical forms $\ddagger$ & $16(30)$ & $636(31.1)$ & $2(25)$ & $14(30.4)$ \\
\hline Silent forms & $7(13)^{\star}$ & $102(5)$ & - & $7(15.3)$ \\
\hline
\end{tabular}

mined by indirect immunofluorescence, using a commercial kit (Endomisio; Eurospital).

Serological HLA typing was performed only in some randomly selected patients (23 with SIgAD and 270 without), as previously described. ${ }^{11}$

The diagnosis of CD was confirmed if the mucosal histology showed subtotal villous atrophy or severe partial villous atrophy with crypt hyperplasia.

Statistical data were generated using SPSS software. The $\chi^{2}$ test with Yates' correction and Fisher's exact test were used for statistical analysis. $p$ values were calculated using the two tailed test. The strength of the association of HLAs was estimated by relative risk (RR), aetiological fraction (EF) and preventive fraction (PF) using methods described elsewhere. ${ }^{12}$ $\mathrm{RR}$ represents the relative risk in contracting a disease in a subject with HLAs compared with a subject without. EF was used when there was a positive association ( $R R>1)$; this measures the contribution of the HLAs to disease susceptibility and, being independent of sample size, gives a clearer estimation of the risk than does RR. PF was calculated when the association was negative $(\mathrm{RR}<1)$; this measures the contribution of a gene to "protection" against disease.

\section{Results}

CLINICAL FINDINGS AT DIAGNOSIS

Of 2098 patients with CD monitored by the 25 centres, 54 with SIgAD were identified (2.6\%); 17 of these had partial IgA deficiency. The

Table 2 Symptoms at diagnosis in patients with selective IgA deficiency (SIgAD) and in those with normal serum IgA (controls)

\begin{tabular}{lll}
\hline & SIgAD & Control \\
\hline Classic forms & $\mathrm{n}=31(\%)$ & $\mathrm{n}=1306(\%)$ \\
Chronic diarrhoea & $29(93.5)$ & $1176(90)$ \\
Weight loss & $29(93.5)$ & $1176(90)$ \\
Abdominal distension & $27(87)$ & $1136(87)$ \\
Vomit & $26(83.9)$ & $1069(81.8)$ \\
Atypical forms & $\mathrm{n}=16(\%)$ & $\mathrm{n}=636(\%)$ \\
Short stature & $6(37.8)$ & $201(31.6)$ \\
Iron deficiency anaemia & $4(25)$ & $213(33.4)$ \\
Increased transferases & $1(6.3)$ & $18(2.8)$ \\
Recurrent abdominal pains with constipation & $1(6.3)$ & $30(4.7)$ \\
Dermatitis herpetiformis & $1(6.3)$ & $31(4.8)$ \\
Recurrent aphthous stomatitis & $3(18.7)$ & $56(8.8)$ \\
Epilepsy and cerebellar calcification & $1(6.3)$ & $11(1.7)$ \\
Miscellaneous & - & $66(10.4)$ \\
Silent forms (serological screening in risk groups) & $\mathrm{n}=7(\%)$ & $\mathrm{n}=102(\%)$ \\
IDDM & $1(14.2)$ & $34(33.3)$ \\
Other autoimmune diseases & - & $6(5.9)$ \\
First degree relatives & $3(42.6)$ & $30(29.4)$ \\
School screening & $1(14.2)$ & $14(13.7)$ \\
SIgAD & $2(28.4)$ & - \\
Down's syndrome & - & $11(10.8)$ \\
Turner's syndrome & - & $2(2)$ \\
Neurological diseases & - & $5(4.9)$ \\
\hline
\end{tabular}

$\star<$ to the third percentile for age.

IDDM, insulin dependent diabetes mellitus. prevalence of SIgAD did not differ in paediatric cases $(46 / 1776,2.6 \%)$ when compared with adult cases $(8 / 322,2.5 \%)$. The prevalence of SIgAD was not influenced by geographical factors, being $22 / 763(2.9 \%)$ in Northern Italy, $14 / 562(2.5 \%)$ in Central Italy, and 18/773 $(2.3 \%)$ in Southern Italy.

At diagnosis, the clinical pattern in patients with SIgAD was different from that in the control patients, with a higher prevalence of the silent form in children (table 1). Symptoms at diagnosis were similar in those with and without SIgAD (table 2), with no differences between adults and children.

In the children, the average age of onset of symptoms was 4.5 years in those with SIgAD and 3.7 years in the controls, while the average age at diagnosis was 6.1 and 5.9 respectively. In the adults, the average age of onset of symptoms was 26 years in those with SIgAD and 24 years in those without, while the average age at diagnosis was 38 and 35 respectively.

Table 3 gives associated diseases: patients with SIgAD had a higher rate of infections and allergic diseases. Autoimmune and malignant diseases were similar in the two groups.

Children with and without SIgAD had similar early feeding habits: $16 / 46(34.7 \%)$ and $570 / 1730(32.9 \%)$ respectively were breast fed for at least three months; in both groups the average age of gluten introduction was at five and a half months.

\section{LABORATORY FINDINGS AT DIAGNOSIS}

At diagnosis, average concentrations of haemoglobin, ferritin, serum iron, calcium, and albumin were similar in the two groups. IgA AGAs and EMAs were absent at diagnosis in all 54 patients with SIgAD, and were present in $1770 / 2044(86.6 \%)$ and $1974 / 2044(96.6 \%)$ of the controls respectively. IgG AGAs were found in $51 / 54(94.4 \%)$ of the patients with SIgAD and in $1819 / 2044(88.9 \%)$ of those with normal IgA values.

At diagnosis, $47 / 54(87 \%)$ of the patients with SIgAD had subtotal villous atrophy (among these were the three subjects without IgG AGAs), and the remainder had severe partial villous atrophy with crypt hyperplasia. In the control group, subtotal villous atrophy was observed in $1675 / 2044(82 \%)$ and severe partial villous atrophy with crypt hyperplasia in $369 / 2044(18 \%)$ of patients.

Serological HLA (class I and II) typing showed differences in the two groups: a higher frequency of DR3 and DQW2 and a lower frequency of B8 antigens were observed in patients with SIgAD than in the control group; table 4 summarises these data.

\section{FOLLOW UP AFTER GFD}

A strict GFD was followed by $1906 / 2098$ patients with CD (52/54 with SIgAD and $1854 / 2044$ with normal values of IgA serum). Similarly, in both groups, within 12-15 months of GFD, the CD symptoms disappeared and an improvement in the "nutritional" laboratory findings (haemoglobin, ferritin, iron serum, calcium, albumin) was observed. In the same 
Table 3 Associated diseases in patients with selective IgA deficiency (SIgAD) and in those with normal serum IgA (controls)

\begin{tabular}{|c|c|c|}
\hline & $\operatorname{SIg} A D(n=54)(\%)$ & Control $(n=2044)(\%)$ \\
\hline Recurrent infections & $16(29.6)$ & $59(2.9)$ \\
\hline Respiratory tract & 14 & 43 \\
\hline Urinary tract & 2 & 3 \\
\hline Gastrointestinal tract & - & 13 \\
\hline Allergic diseases & $7(13)$ & $95(4.6)$ \\
\hline Asthma & 2 & 28 \\
\hline Atopic dermatitis & 3 & 23 \\
\hline Gastrointestinal allergy & 2 & 6 \\
\hline Chronic urticaria & - & 6 \\
\hline Allergic rhinitis & - & 28 \\
\hline Allergy to drugs & - & 4 \\
\hline Autoimmune diseases & $3(5.5)$ & $111(5.4)$ \\
\hline Insulin dependent diabetes mellitus (IDDM) & 2 & 86 \\
\hline IDDM + autoimmune thyroiditis & - & 1 \\
\hline Autoimmune thyroiditis + autoimmune hepatitis & _- & 2 \\
\hline Autoimmune hepatitis & - & 3 \\
\hline Scleroderma & - & 1 \\
\hline Thrombocytopenic purpura & - & 1 \\
\hline Arthritis & - & 5 \\
\hline Uveitis & 1 & - \\
\hline Thyroiditis & - & 4 \\
\hline Autoimmune polyendocrinopathy & - & 1 \\
\hline Miscellaneous & - & 7 \\
\hline Malignant diseases & - & $5(0.2)$ \\
\hline Burkitt's lymphoma & - & 1 \\
\hline Intestinal lymphoproliferative disease & - & 1 \\
\hline Leukaemia & - & 1 \\
\hline Medulloblastoma & - & 1 \\
\hline Waldestrom blastoma & - & 1 \\
\hline Chromosomal diseases & - & $22(1.1)$ \\
\hline Down's syndrome & - & 16 \\
\hline Down's syndrome + IDDM & - & 1 \\
\hline $\begin{array}{l}\text { Down's syndrome }+ \text { autoimmune thyroiditis }+ \text { autoimmune hepatitis }+ \\
\text { myelomonocytic leukaemia }\end{array}$ & - & 1 \\
\hline Turner's syndrome & - & 3 \\
\hline Turner's syndrome + IDDM & - & 1 \\
\hline Neurological diseases & $1(1.9)$ & $17(0.8)$ \\
\hline Epilepsy and cerebellar calcification & 1 & 11 \\
\hline Epilepsy & - & 3 \\
\hline Psycomotor encephalopathy & - & 3 \\
\hline Other associations & $6(11.1)$ & $205(10)$ \\
\hline Dermatitis herpetiformis & 1 & 31 \\
\hline Increase of transaminases & 1 & 101 \\
\hline Dental enamel hypoplasia & 1 & 10 \\
\hline Psoriasis & - & 3 \\
\hline Infertility & - & 4 \\
\hline Aphthous stomatitis & 3 & 56 \\
\hline
\end{tabular}

Recurrent infections: SIgAD $v$ control $\mathrm{p}<0.00001$; allergic diseases: SIgAD $v$ control $\mathrm{p}<0.02$.

period AGAs and EMAs, if initially high, returned to normal in almost all patients: $47 / 52$ $(90.4 \%)$ of those with SIgAD showed IgG AGA reduction, while in the control group normalisation of IgA AGAs, IgG AGAs, and EMAs was observed in $1780 / 1854(96.1 \%)$, $1668 / 1854(89.9 \%)$, and $1816 / 1854(97.9 \%)$ of the patients respectively. Seven of the patients with SIgAD (five with partial IgA serum deficiency; one adult and four children) showed normalisation of serum IgA levels after 36-48 months of GFD.

After 12-15 months of a strict GFD, a biopsy was performed in $16 / 52$ of the IgA deficient patients and 685/1854 patients of the control group. Mucosal improvement was observed in all cases.

Table 4 HLA typing in patients with selective IgA deficiency (SigAD) and in those with normal serum IgA (controls)

\begin{tabular}{llllll}
\hline & SigAD $(n=23)(\%)$ & Control $(n /$ total $)(\%)$ & $E F$ & $P F$ & $R R$ \\
\hline B8 & $4(17.4)$ & $70 / 174(40.2)$ & - & 0.28 & 0.31 \\
DR3 & $21(91.3)$ & $130 / 270(48.1)$ & 0.83 & - & 11.31 \\
DR7 & $12(52.2)$ & $96 / 270(35.5)$ & 0.26 & - & 1.98 \\
DQW2 & $22(95.7)$ & $217 / 272(79.7)$ & 0.79 & - & 5.58 \\
\hline
\end{tabular}

$\star \operatorname{SIgAD} v$ control $\mathrm{p}<0.002$.

$\mathrm{RR}$, relative risk; $\mathrm{EF}$, aetiological fraction; $\mathrm{PF}$, preventive fraction.

\section{Discussion}

Our study shows that IgA deficient subjects are at risk for CD. The prevalence of SIgAD in the general population ranges from 1:500 $(0.2 \%)$ to $1: 700(0.14 \%),{ }^{13}$ whereas in patients with $\mathrm{CD}$ it is $2.6 \%$. Our data should represent the national situation because a large number of patients were examined (2098), who were evenly distributed across Italy.

As previously reported by others, ${ }^{1-3} \mathrm{CD}$ symptoms at presentation were similar in patients with or without SIgAD, but it is interesting that we observed a higher prevalence of silent forms in those with SigAD. This is in accordance with a previous study ${ }^{4}$ and suggests that subjects with SigAD should be screened for CD, even if they do not have evident symptoms. The silent forms were observed more frequently in children than in adults; this may be the result of clinical practices because gastroenterologists examining adult patients are not as often looking for these forms of CD as are paediatricians.

In contrast with other studies, ${ }^{134}$ our study shows that patients with SIgAD are more susceptible to infections and allergic diseases than those without. This may be explained by 
the much higher total number of subjects in our study, which should be enough to show that the prevalence of infection and allergic diseases is as we observed.

A similar incidence of autoimmune disease was found in both patients with SIgAD and the control group. This could be due to a bias depending on age at diagnosis and to the duration of gluten exposure: in patients with CD the predisposition to autoimmune disease is related to both of these factors, ${ }^{14}{ }^{15}$ and in all our cases (both those with SIgAD and those without) the average age at diagnosis was low and the duration of gluten exposure the same.

An increased risk of malignancy has been established in both $\mathrm{CD}^{16}$ and SigAD-CD, ${ }^{9}$ but we did not find a higher incidence in our groups. This may be explained by the low average age of our patients at diagnosis and by their adhesion to a GFD, because patients on a strict precocious GFD do not run any increased risk of malignancy. ${ }^{16}$

It is possible that SIgAD predisposes to $\mathrm{CD}$ because it leads to insufficient exclusion of food antigens. However, the early feeding practices (breast feeding and age of gluten introduction) were similar in all our paediatric patients (both those with SIgAD and the control group); this suggests that these environmental factors do not predispose to gluten intolerance in IgAD. SIgAD and CD, on the other hand, share a significantly increased correlation $^{17}$ with certain HLAs (B8, DR3, DR7, DQW2). This was confirmed in our patients: those with SigAD showed a closer correlation with DR3 $(\mathrm{p}=0.00191 ; \mathrm{RR}=$ $11.31, \mathrm{EF}=0.83)$ and $\mathrm{DQW} 2(\mathrm{RR}=5.58, \mathrm{EF}$ $=0.79)$ than the control group. These findings, which are in some respect very similar to those reported by a previous study, ${ }^{18}$ suggest that CD and SIgAD may share predisposing genes and that some HLAs may be involved in triggering the onset of gluten intolerance in SIgAD. The laboratory findings were similar for the patients with SIgAD and the control group, both at diagnosis and at follow up, except that the former did not produce IgA AGAs and EMAs. Consequently, it is probably advisable to measure serum IgA concentration routinely in serological screening for CD. In our series, 3/54 patients with SIgAD had normal levels of IgG AGA: two underwent an intestinal biopsy because they had symptoms suggesting CD, and the third (who was symptomless) simply because he was at risk for CD (first degree relative of a patient with $\mathrm{CD}$ ). For this reason, and because the assay for serum $\operatorname{IgA}$ is not expensive, serological screening for CD should include the routine measurement of serum IgA.

In conclusion, the association of SIgAD with $\mathrm{CD}$ is significantly more frequent than in the general population, in both adults and children. Silent forms of CD are more common in those with SIgAD than in those without, and the former are more likely to suffer from atopic diseases and recurrent infections. Furthermore, since most patients with CD and SIgAD lack IgA EMAs, and some also lack IgG AGAs, intestinal biopsy should be performed in all cases of $\operatorname{IgA}$ deficiency where there is a suspicion of CD.

The SIGEP and the Club del Tenue working groups on coeliac diseases are comprised of: Ancona, Clinica Pediatrica (C Catassi, E Fabiani); Bari, Clinica Pediatrica 1 (R Baldassarre); Bologna I, Clinica Pediatrica 3 (R Lazzari); Bologna II, Ospedale Maggiore, 2nd Divisione di Pediatria (M G Zaniboni, A Lambertini); Brescia, Clinica Pediatrica (A Meini, A G Ugazio); Catania, Clinica Pediatrica (G Bottaro); Catanzaro, Clinica Pediatrica (S Guandalini, L Pensabene); Chieti, Clinica Pediatrica (F Bascietto); Imola, Ospedale della Scaletta Pivisione di Pediatria (S Brusa); L'Aquila, Dipartimento di Divisione di Pediatria (S Brusa); L’Aquila, Dipartimento di IV (M Fontana); Milano II, Clinica Pediatrica 2 (A M Giunta, IV (M Fontana); Milano II, Clinica Pediatrica 2 (A M Giunta,
LPrampolini); Milano III, Clinica Pediatrica 3 (C Bianchi, G LPrampolini); Milano III, Clinica Pediatrica 3 (C Bianchi, G
Barera); Modena, Clinica Pediatrica (F Balli, P Bertolani); Barera); Modena, Clinica Pediatrica (F Balli, P Bertolani);
Padova, Clinica Pediatrica (G Guariso); Palermo, Clinica Pediatrica 2 P.O. Aiuto Materno (F Cataldo, P Distefano, V Marino, $M$ Violante, G Traverso); Perugia,Cattedra di Gastroenterologia ( A M Pelli); Pisa, Clinica Pediatrica (A Ventura, C Ughi); Roma I, Clinica Pediatrica, Universita' La Sapienza (F Viola, M Barbato); Roma II, Servizio Dietologia Ospedale Bambin Gesu' (A M Ambruzzi); Napoli I, Cattedra di Gastroenterologia (C Ciacci); Napoli II, Clinica Pediatrica III (F Rea); S. Giovanni Rotondo, Casa del Sollievo e della Sofferenza, Divisione di Pediatria (M R D'Altilia); Torino I, Clinica Pediatrica (N Ansaldi Balocco, L Bramante); Torino II, Cattedra di Gastroenterologia (C Sabra Guidetti); Trieste, Clinica PediatricaIstituto Burlo Garofalo per l'Infanzia (G Torre, S Martellossi).

1 Collin P, Maki M, Keyrilainen O, et al. Selective IgA deficiency and coeliac disease. Scand $\mathcal{f}$ Gastroenterol 1992;27:367-71.

2 Meini A, Pillan NM, Villanacci V, et al. Prevalence and diagnosis of coeliac disease in IgA-deficient children. Ann nosis of coeliac disease in IgA-deficie
Allergy Asthma Immunol 1996;77:333-6.

Allergy Asthma Immunol 1996;77:333-6.
Savilahti E, Pelkonen P, Verkasalo M, Koskimies S. Selective Savilahti E, Pelkonen P, Verkasalo M, Koskimies S. Selective
deficiency of immunoglobulin A. Klin Padiatr 1985;197: 336-40.

4 Klemola T. IgA deficiency. Annals of Clinical Research 1987; 19:248-57.

5 Mawhinney H, Tomkin GH. Gluten enteropathy associated with selective IgA deficiency. Lancet 1971;2:121-4.

6 Savilahti E, Pelkonen P, Visakorpi JK. IgA deficiency in children. A clinical study with special reference to intestinal findings. Arch Dis Child 1971;46:665-70.

7 Meuwisse GH. Diagnostic criteria in coeliac disease. Acta Paediatr 1970;59:461-3.

8 Working Group of ESPGAN. Revised criteria for diagnosis of coeliac disease. Arch Dis Child 1990;65:909-11.

9 De Laat PCJ, Weemaes CMR, Gonera R, et al. Clinical De Laat PCJ, Weemaes CMR, Gonera R, et al. Clinical
manifestations in selective IgA deficiency in childhood. A manifestations in selective IgA deficiency in child
follow-up report. Acta Paediatr 1991;80:798-804.

10 Ritchie RF. Automated quantification of proteins in serum and other biological fluids. Am F Clin Pathol 1973;59:1517.

11 Van Rod JJ. Microlimphocytotoxicity method. In: Manual of tissue typing techniques. Bethesda, MD: DHEV, 1979:104112.

12 Green A. The epidemiologic approach to studies of association between HLA and disease. I. The basic measures, concepts and estimation procedures. Tissue Antigens 1982; 19:245-58.

13 De Martino M, Gallo L, De Luca M, et al. Biologia e clinica del deficit di IgA in pediatria. Rivista Italiana di Infettivologia Pediatrica 1992;7:7-16.

14 Pocecco M, Ventura A. Coeliac disease and insulin dependent diabetes mellitus: a casual association? Acta Paediatr 1995;84:1432-3.

15 Ventura A, Magazzu' G. Autoimmune disorders in coeliac disease. Seventh International Symposium on Coeliac Disease. disease. Seventh International Symposium

16 Holmes GKT. Long-term health risks for unrecognized coeliac patients. In: Auricchio S, Visakorpi JK, eds. Common food intolerance: epidemiology of coeliac disease. Basel: Karger, $1992 ; 105-18$

17 Clerici N, Fernandez M, Saiz I, Polanco I. Human leucocyte antigen alleles and aplotypes associated with selective mmunoglobulin A deficiency in Spanish paediatric patients. F Pediatr Gastroenterol Nutr 1993;16:381-6

18 Klemola T, Savilahti E, Koskimies S, Pelkonen P. HLA antigens in IgA deficient paediatric patients. Tissue Antigens 1988;32:218-23. 